\section{Desigualdades sócio-espaciais da adequação das informações de nascimentos e óbitos do Ministério da Saúde, Brasil, 2000-2002}

\author{
Socio-spatial inequalities in the adequacy of \\ Ministry of Health data on births and deaths at \\ the municipal level in Brazil, 2000-2002
}

\author{
${ }^{1}$ Escola Nacional de Saúde \\ Pública Sergio Arouca, \\ Fundação Oswaldo Cruz, \\ Rio de Janeiro, Brasil. \\ 2 Centro de Informação \\ Científica e Tecnológica, \\ Fundação Oswaldo Cruz, \\ Rio de Janeiro, Brasil. \\ Correspondência \\ C. L. T. Andrade \\ Departamento de \\ Administração e \\ Planejamento em Saúde, \\ Escola Nacional de Saúde \\ Pública Sergio Arouca, \\ Fundação Oswaldo Cruz. \\ Rua Leopoldo Bulhões 1480 , \\ Rio de Janeiro, $R J$ \\ 21041-210, Brasil. \\ carlamv@ensp.fiocruz.br
}

\begin{abstract}
This study analyzed socio-spatial inequalities in the adequacy of Ministry of Health data systems on live births (SINASC) and deaths (SIM) for estimating infant mortality at the municipal level in Brazil. Data from 2000-2002 for all $\mathrm{mu}$ nicipalities were analyzed according to population size and geographic region. Five indicators were considered: age-standardized mortality rate; ratio of reported-to-estimated live births; relative mean deviation of the mortality rate; relative mean deviation of the birth rate; and proportion of deaths with undetermined causes. Adequacy criteria were established statistically for eight Brazilian States in which vital statistics were adequate. The results showed important socio-spatial inequalities: in general, the proportion of adequate vital statistics was higher in the Central-South of the country and in larger municipalities. The live birth data system received the best evaluation for three items. The mortality data system requires both a reduction in underreporting and improved data on cause of death in order to orient health programs focused on decreasing inequalities in infant mortality in Brazil.
\end{abstract}

Information Systems; Infant Mortality; Indicators
Carla Lourenço Tavares de Andrade 1 Célia Landmann Szwarcwald 2

\section{Introdução}

Em virtude da estreita relação entre a mortalidade infantil e as condições sócio-econômicas e de saúde de uma população, o coeficiente de mortalidade infantil tem sido amplamente utilizado para sintetizar e comparar a situação de saúde dos países. Entretanto, apesar do uso consagrado desse indicador de saúde, a maioria das nações em desenvolvimento não dispõe de dados para a sua estimativa com grau satisfatório de confiabilidade. Definido como o número de óbitos infantis para cada mil nascidos vivos, o cálculo direto do coeficiente de mortalidade infantil depende da completitude das informações de registro de óbitos e nascimentos.

A dificuldade de obtenção de estimativas fidedignas da mortalidade infantil devido às limitações nas fontes primárias de informações estimulou a elaboração de procedimentos metodológicos para a sua estimação. A partir de meados do século XX, a pesquisa demográfica dedicou-se à formulação de técnicas de estimação indireta desse indicador, de forma a substituir as estimativas diretas, passíveis de graves erros em situações de coberturas incompletas das estatísticas vitais 1 .

Os métodos de mensuração indireta, aperfeiçoados ao longo do tempo e reconhecidos pela robustez, têm sido amplamente utilizados para fornecer o nível da mortalidade infantil nos países com grande precariedade de registro de 
nascimentos e óbitos. A partir da década de 70 , em grande parte dos países da América Latina, tornou-se habitual incluir perguntas de caráter retrospectivo nos censos e pesquisas domiciliares, destinadas especificamente à mensuração indireta da mortalidade 2,3.

Entretanto, as estimativas obtidas por técnicas de mensuração indireta dependem da realização de censos demográficos e pesquisas por amostragem. Não tendo o caráter de continuidade, peculiar aos dados de registro, a mensuração indireta da mortalidade infantil restringe o monitoramento contínuo desse indicador, dificultando a avaliação imediata da efetividade de ações e programas de saúde dirigidos, especificamente, às crianças menores de um ano de idade. Adicionalmente, as estimativas elaboradas a partir de pesquisas por amostragem, devido às limitações no tamanho da amostra, não podem ser fornecidas em distintos níveis de desagregação geográfica, o que não permite identificar os municípios com maiores problemas na situação de saúde infantil.

Um problema adicional encontra-se na diversidade dos resultados obtidos por diferentes procedimentos de estimação. Seja utilizando-se técnicas de mensuração indireta a partir de dados de censos ou pesquisas por amostragem, seja corrigindo as informações de registro, dependendo do método utilizado, as estimativas são díspares, dificultando a interpretação da tendência e o conhecimento do nível verdadeiro da mortalidade infantil na população estudada 4 .

No caso particular do Brasil, o Ministério da Saúde dispõe, atualmente, de dois sistemas de informação em saúde, cujos dados são apropriados para o cálculo do coeficiente de mortalidade infantil: o Sistema de Informações sobre Mortalidade (SIM) e o Sistema de Informações sobre Nascidos Vivos (SINASC). As informações são disponibilizadas na Internet, no nível de município (http://www.datasus.gov.br). O reconhecimento da importância de monitoramento das informações sobre óbitos e nascimentos junto à facilidade de acesso aos dados têm resultado no aumento substancial na cobertura e na qualidade das informações de ambos os sistemas.

Entretanto, a persistente precariedade dos dados em alguns Estados, sobretudo os localizados nas regiões Norte e Nordeste, ainda não permite o cálculo direto da mortalidade infantil, em âmbito nacional, acarretando na utilização das estimativas elaboradas pelo Instituto Brasileiro de Geografia e Estatística (IBGE), órgão responsável pela estimação da mortalidade infantil no país. Tais estimativas são baseadas em dados dos censos populacionais e das Pesquisas Nacionais por Amostras de Domicílios (PNAD) e calculadas por métodos demográficos indiretos. Comparadas às estimativas realizadas em estados e municípios de capitais, onde as coberturas das informações de óbitos são, reconhecidamente, completas, têm mostrado inconsistências relevantes 5 .

Alguns critérios de avaliação da adequação das informações do SIM e do SINASC foram propostos previamente por Szwarcwald et al. 5 . Seguindo sugestões de vários pesquisadores e gestores do setor saúde após a utilização da metodologia proposta, os critérios foram aperfeiçoados e usados no presente estudo, com o objetivo de investigar as desigualdades sócio-espaciais da adequação das informações de nascimentos e óbitos do Ministério da Saúde para o cálculo da mortalidade infantil no Brasil.

\section{Material e métodos}

A análise das informações de óbitos e nascimentos do Ministério da Saúde foi realizada para todos os municípios brasileiros no período de 2000 a 2002. As fontes de informações utilizadas foram o SIM, o SINASC, além da base de população residente por município (http://www.datasus. gov.br).

A seguir, listam-se os indicadores usados para estabelecer os critérios de adequação das informações vitais dos municípios.

\section{Coeficiente geral de mortalidade padronizado por idade}

Define-se o coeficiente geral de mortalidade (CGM) como sendo o número total de óbitos por mil habitantes em determinado ano e lugar. Por entender que as estruturas etárias das populações diferem por município, o CGM foi padronizado por idade. A população padrão foi a do Estado do Rio de Janeiro no ano de 2000, por ser o estado que apresenta a maior proporção de idosos (60 anos e mais) para o triênio 2000-2002.

\section{Desvio médio relativo do CGM}

Este indicador é definido como a razão entre o desvio médio do CGM nos anos 2000, 2001 e 2002 em relação ao coeficiente geral de mortalidade médio do período:

DMCGM $=\frac{\left|\mathrm{CGM}_{00}-\mathrm{CGM}_{\text {med }}\right|+\left|\mathrm{CGM}_{01}-\mathrm{CGM}_{\text {med }}\right|+\left|\mathrm{CGM}_{02}-\mathrm{CGM}_{\text {med }}\right|}{3 \times \mathrm{CGM}_{\text {med }}}$,

Onde, $\mathrm{CGM}_{00}=\mathrm{CGM}$ no ano de 2000; $\mathrm{CGM}_{01}$ = CGM no ano de 2001; $\mathrm{CGM}_{02}=\mathrm{CGM}$ no ano de 2002; $\mathrm{CGM}_{\text {med }}=\mathrm{CGM}$ no período 2000-2002.

Sabendo-se que não há grandes alterações no CGM de uma população em um período curto de três anos, este indicador pode ser utilizado 
para avaliar a regularidade das informações de óbitos.

\section{Razão entre nascidos vivos}

informados e estimados

O número de nascidos vivos pode ser estimado pela população de menores de um ano, considerada como a coorte de nascidos vivos que sobreviveram. Supondo-se uma taxa de mortalidade infantil $q_{0}$, o número de nascidos vivos é estimado por:

População de menores de um ano

1- $\left(0,5 * \mathrm{q}_{0}\right)$

O indicador é calculado pela razão entre o número de nascidos vivos informados no SINASC e o número de nascidos vivos estimado pela população de menores de um ano. Valores baixos da razão, indicam subenumeração de nascidos vivos no SINASC.

\section{Desvio médio relativo da taxa de natalidade}

Da mesma forma que a mortalidade, é esperado que não haja grandes alterações na taxa de natalidade em um período de três anos. Expressando a flutuação no número informado de nascidos vivos, esse indicador é construído de maneira análoga ao desvio médio do CGM, sendo definido pela razão entre o desvio médio das taxas de natalidade nos anos 2000, 2001 e 2002 em relação à taxa média de natalidade no período.

\section{Proporção de óbitos sem}

definição da causa básica

A proporção de óbitos classificados na rubrica "sintomas, sinais e achados anormais de exames clínicos e de laboratório não classificados em outra parte" representa a fração das mortes em que não houve definição da causa básica de óbito. $\mathrm{O}$ uso desse indicador na análise justifica-se por expressar irregularidades na qualidade da notificação das informações de óbitos.

Dos 5.561 municípios brasileiros, 54 foram criados no ano de 2001, e, por conseguinte, não foram considerados no estudo. A análise foi realizada de acordo com a categoria de porte populacional do município: menos de 50 mil habitantes; 50 mil habitantes ou mais.

Para definir critérios de adequação das informações vitais, foram analisadas as Unidades da Federação (UF) com informações consideradas adequadas pela Rede Interagencial de Informações para a Saúde (RIPSA) 6 - Espírito Santo, Rio de Janeiro, São Paulo, Paraná, Santa Catarina, Rio Grande do Sul, Mato Grosso do Sul e Distrito Federal.
Inicialmente, os municípios localizados nessas oito UF foram categorizados em dois grupos: menos de 50 mil habitantes; 50 mil habitantes ou mais. Com as informações dos municípios do primeiro grupo, foram estabelecidos os critérios de adequação para todos os municípios brasileiros com menos de 50 mil habitantes, enquanto com as do segundo grupo, foram estabelecidos os critérios de adequação dos municípios brasileiros com 50 mil ou mais habitantes. A seguir, em cada um dos dois grupos de municípios das oito UF, foram calculados as médias e limites de confiança dos cinco indicadores utilizados para qualificar as informações de óbitos e nascimentos.

Os critérios de definição de "satisfatório" foram, então, estabelecidos por categoria populacional (menos de 50 mil habitantes; 50 mil habitantes ou mais), tomando como base os limites unilaterais de confiança de 90\% de cada indicador. Por sua vez, os critérios de definição de "deficiente" foram estabelecidos utilizandose os limites unilaterais de $99,9 \%$ de confiança, ou seja, considerando a fração $0,1 \%$ fora da normalidade. Os critérios de definição de "não satisfatório" foram os limites intermediários entre as classificações "satisfatório" e "deficiente". O resumo desses critérios está apresentado na Tabela 1.

De acordo com os critérios estabelecidos, todos os municípios foram classificados em satisfatório, não satisfatório ou deficiente para cada dimensão avaliada - "Mortalidade", "Natalidade" e "Mal Definidos" - sendo utilizados os seguintes indicadores, respectivamente, para cada aspecto: CGM padronizado e desvio médio dos óbitos; razão entre nascidos vivos informados e estimados e desvio médio dos nascimentos; proporção de óbitos sem definição da causa básica. A partir dessa classificação, o município foi considerado como tendo: (I) informações vitais satisfatórias - quando todos os critérios são satisfatórios em todas as três dimensões avaliadas; (II) informações vitais não satisfatórias - quando pelo menos um dos aspectos não é satisfatório e nenhum é deficiente; (III) informações vitais deficientes - quando pelo menos um dos aspectos é deficiente.

A análise da adequação das informações por município do Brasil foi realizada baseando-se nas diferenças sócio-espaciais de adequação das informações por macrorregião geográfica e tamanho de população do município.

A visualização da distribuição geográfica dos municípios segundo a adequação das informações vitais foi realizada por meio de mapas temáticos usando o aplicativo MapInfo (MapInfo Corporation, Nova York, Estados Unidos). 
Critérios * para classificação dos municípios segundo a adequação das informações vitais por porte populacional. Brasil, $2000-2002$.

\begin{tabular}{|c|c|c|c|c|c|c|}
\hline \multirow[t]{2}{*}{ Indicador } & \multicolumn{3}{|c|}{ Municípios menores de 50 mil habitantes } & \multicolumn{3}{|c|}{ Municípios com 50 mil habitantes } \\
\hline & Satisfatório & Não satisfatório & Deficiente & Satisfatório & Não satisfatório & Deficiente \\
\hline Coeficiente geral de mortalidade padronizado por idade & $\geq 5,0$ & $\geq 3,1$ e $<5,0$ & $<3,1$ & $\geq 6,3$ & $\geq 4,8$ e $<6,3$ & $<4,8$ \\
\hline Razão entre nascidos vivos informados e estimados & $\geq 0,7$ & $\geq 0,5$ e $<0,7$ & $<0,5$ & $\geq 0,9$ & $\geq 0,8$ e $<0,9$ & $<0,8$ \\
\hline Proporção de óbitos mal definidos & $\leq 20,7$ & $\leq 35,7$ e $>20,7$ & $>35,7$ & $\leq 16,2$ & $\leq 27,4$ e $>16,2$ & $>27,4$ \\
\hline Desvio médio relativo da taxa de natalidade & $\leq 17,1$ & $\leq 28,6$ e $>17,1$ & $>28,6$ & $\leq 8,1$ & $\leq 11,9$ e $>8,1$ & $>11,9$ \\
\hline Desvio médio relativo do coeficiente geral de mortalidade & $\leq 20,9$ & $\leq 35,1$ e $>20,9$ & $>35,1$ & $\leq 6,1$ & $\leq 10,4$ e $>6,1$ & $>10,4$ \\
\hline
\end{tabular}

* Obtidos a partir da análise das informações de oito Unidades da Federação (Espírito Santo, Rio de Janeiro, São Paulo, Paraná, Santa Catarina, Rio Grande do Sul, Mato Grosso do Sul e Distrito Federal), consideradas com informações adequadas 6 .

\section{Resultados}

Na Tabela 2 é apresentada a distribuição percentual dos municípios de acordo com a adequação das informações vitais segundo Grande Região. Dos municípios brasileiros, 90,3\% deles têm menos de 50 mil habitantes. Nesse grupo de municípios, considerando-se as três dimensões ("Mortalidade", "Natalidade" e "Mal Definidos"), conjuntamente, é nítido o gradiente de desigualdade entre as regiões: o percentual de municípios que têm grau satisfatório varia de $5 \%$, no Nordeste, a $63 \%$, no Sul. Já quando cada dimensão é considerada separadamente, a maior variação regional corresponde à definição da causa de óbito, seguida pela adequação das informações de mortalidade. Chama a atenção que somente $15 \%$ dos municípios de pequeno porte da região Nordeste têm informações adequadas quanto à definição da causa básica de morte.

Analisando os resultados relativos aos municípios com 50 mil habitantes ou mais, apresentados na Tabela 2, os percentuais de municípios com informações satisfatórias são um pouco maiores, embora persistam as desigualdades regionais em nível bem acentuado. Considerando as três dimensões avaliadas, o percentual de municípios com deficiência de informações alcança $63 \%$ na Região Nordeste, contrapondo com 3\% na Região Sul.

Os dados apresentados na Tabela 3 mostram que pouco mais de um terço da população brasileira vive em municípios com menos de 50 mil habitantes, embora estes correspondam a $90 \%$ dos municípios brasileiros.

No que se refere à adequação das informações, no total do Brasil, cerca de $54 \%$ da população vivem em municípios que têm grau satisfatório na informação dos eventos vitais. Percebe-se que as desigualdades ocorrem tanto por porte populacional como por macrorregião geográfica. Esse percentual varia de $40 \%$, na categoria de municípios pequenos, a $62 \%$, no grupo de municípios de maior porte. Da mesma forma, os percentuais de adequação estão no patamar de $20 \%$ no Norte e Nordeste, mas são superiores a $65 \%$ no Centro-Sul. Observa-se também que as diferenças regionais persistem dentro de cada categoria de tamanho de município (Tabela 3).

Na Figura 1 é mostrada a distribuição espacial dos municípios brasileiros de acordo com a adequação das informações vitais de óbitos e nascimentos. Por meio da Figura la, observa-se que a maioria dos municípios classificados com grau satisfatório para a dimensão relativa à mortalidade encontra-se nas regiões Sudeste, Sul e CentroOeste, enquanto os com classificação deficiente predominam nas regiões Norte e Nordeste. Distribuição geográfica similar é apresentada para a proporção de óbitos mal definidos (Figura 1b), ainda com maiores contrastes regionais. Pela Figura 1c, a dimensão referente às informações de natalidade mostra uma distribuição mais equânime, já que os municípios classificados como satisfatórios são encontrados em toda a extensão do território brasileiro.

Na Figura 1d, considerando-se as três dimensões em conjunto, observa-se a influência da adequação das informações de mortalidade e da proporção de óbitos mal definidos, obtendose os mesmos contrastes entre o Centro-Sul e o Norte-Nordeste.

\section{Discussão}

No Brasil, o SIM vem demonstrando nítidos avanços, seja no que se refere à ampliação da cobertura, seja na divulgação e facilidade de acesso aos dados. A oportunidade de examinar as in- 
Distribuição percentual (\%) dos municípios de acordo com a adequação das informações vitais por dimensão avaliada segundo porte populacional e Grande Região. Brasil, 2000-2002.

\begin{tabular}{|c|c|c|c|c|c|c|c|c|c|c|c|c|c|c|}
\hline & \multirow[t]{3}{*}{$\mathbf{n}$} & \multirow{3}{*}{$\begin{array}{l}\text { Percentual } \\
\text { do total de } \\
\text { municípios }\end{array}$} & \multicolumn{12}{|c|}{ Dimensão avaliada } \\
\hline & & & \multicolumn{3}{|c|}{ Mortalidade } & \multicolumn{3}{|c|}{ Natalidade } & \multicolumn{3}{|c|}{ Mal definidos } & \multicolumn{3}{|c|}{3 dimensões } \\
\hline & & & I & II & III & I & II & III & 1 & II & III & I & II & III \\
\hline \multicolumn{15}{|c|}{ Municípios menores de } \\
\hline \multicolumn{15}{|c|}{50 mil habitantes } \\
\hline Norte & 404 & 7,3 & 23,5 & 44,8 & 31,7 & 54,7 & 28,0 & 17,3 & 38,4 & 24,5 & 37,1 & 6,4 & 33,9 & 59,7 \\
\hline Nordeste & 1.643 & 29,8 & 37,7 & 46,5 & 15,8 & 71,2 & 20,5 & 8,3 & 14,5 & 20,6 & 64,8 & 4,9 & 24,1 & 71,0 \\
\hline Sudeste & 1.446 & 26,3 & 68,7 & 22,7 & 8,6 & 73,7 & 19,6 & 6,8 & 69,6 & 18,9 & 11,5 & 47,5 & 32,4 & 20,1 \\
\hline Sul & 1.066 & 19,4 & 81,5 & 16,4 & 2,1 & 81,8 & 15,9 & 2,3 & 90,2 & 9,0 & 0,8 & 63,3 & 32,0 & 4,7 \\
\hline Centro-Oeste & 416 & 7,6 & 59,9 & 33,9 & 6,3 & 73,6 & 21,9 & 4,6 & 89,7 & 8,4 & 1,9 & 47,4 & 41,1 & 11,5 \\
\hline Brasil & 4.975 & 90,3 & 56,8 & 31,9 & 11,3 & 73,0 & 20,0 & 7,0 & 55,0 & 16,9 & 28,1 & 33,5 & 30,4 & 36,1 \\
\hline \multicolumn{15}{|c|}{ Municípios com 50 mil } \\
\hline \multicolumn{15}{|c|}{ habitantes ou mais } \\
\hline Norte & 45 & 0,8 & 31,1 & 31,1 & 37,8 & 48,9 & 26,7 & 24,4 & 33,3 & 20,0 & 46,7 & 15,6 & 26,7 & 57,8 \\
\hline Nordeste & 144 & 2,6 & 32,6 & 40,3 & 27,1 & 63,9 & 18,1 & 18,1 & 25,7 & 26,4 & 47,9 & 9,0 & 28,5 & 62,5 \\
\hline Sudeste & 220 & 4,0 & 79,5 & 17,3 & 3,2 & 82,7 & 14,5 & 2,7 & 83,6 & 14,5 & 1,8 & 57,7 & 35,9 & 6,4 \\
\hline Sul & 93 & 1,7 & 78,5 & 21,5 & 0,0 & 73,1 & 23,7 & 3,2 & 93,5 & 6,5 & 0,0 & 53,8 & 43,0 & 3,2 \\
\hline Centro-Oeste & 30 & 0,5 & 66,7 & 26,7 & 6,7 & 76,7 & 16,7 & 6,7 & 93,3 & 6,7 & 0,0 & 56,7 & 33,3 & 10,0 \\
\hline Brasil & 532 & 9,7 & 61,8 & 25,9 & 12,2 & 72,7 & 18,2 & 9,0 & 66,0 & 16,4 & 17,7 & 40,2 & 34,2 & 25,6 \\
\hline \multicolumn{15}{|c|}{ Total de municípios } \\
\hline Norte & 449 & 8,2 & 24,3 & 43,4 & 32,3 & 54,1 & 35,0 & 10,9 & 37,9 & 24,1 & 38,1 & 7,3 & 34,5 & 58,1 \\
\hline Nordeste & 1.787 & 32,4 & 37,3 & 46,0 & 16,7 & 70,6 & 21,8 & 7,6 & 15,4 & 21,1 & 63,5 & 5,3 & 24,5 & 70,2 \\
\hline Sudeste & 1.666 & 30,3 & 70,2 & 22,0 & 7,9 & 74,8 & 19,5 & 5,6 & 71,5 & 18,3 & 10,2 & 48,8 & 33,3 & 17,9 \\
\hline Sul & 1.159 & 21,0 & 81,3 & 16,8 & 1,9 & 81,1 & 16,9 & 2,0 & 90,4 & 8,8 & 0,8 & 62,6 & 33,2 & 4,2 \\
\hline Centro-Oeste & 446 & 8,1 & 60,3 & 33,4 & 6,3 & 73,8 & 22,6 & 3,6 & 89,9 & 8,3 & 1,8 & 48,0 & 41,7 & 10,3 \\
\hline Brasil & 5.507 * & 100,0 & 57,3 & 31,4 & 11,3 & 73,0 & 19,8 & 7,2 & 56,0 & 16,9 & 27,1 & 34,1 & 30,8 & 35,1 \\
\hline
\end{tabular}

* 54 municípios foram excluídos da análise por terem sido criados em 2001.

I: satisfatório; II: não satisfatório; III: deficiente.

formações de óbitos e nascimentos em todos os municípios brasileiros abriu possibilidades para analisar a qualidade da informação e identificar irregularidades locais, passíveis de melhora com o tempo.

Trabalhos de investigação sobre a evolução temporal da mortalidade infantil mostram que houve decréscimo relevante em todo o território nacional, persistindo, porém, as disparidades regionais 7 . A diminuição mais expressiva ocorreu para o período pós-neonatal, devido, principalmente, à redução das mortes por doenças infecciosas intestinais e infecções respiratórias agudas ${ }^{8}$. A redução relevante das doenças preveníveis por imunização e a diminuição da desnutrição - implicando um decréscimo das mortes por crescimento fetal retardado e por má nutrição fetal - também foram nítidas no processo de transição da situação de saúde entre os menores de um ano ${ }^{9}$.
Entretanto, a despeito dos avanços conseguidos, existe ainda um grande espaço para a redução da mortalidade infantil no Brasil. Medidas que podem determinar grande impacto sobre as causas perinatais - o agrupamento mais importante - podem ser implementadas na esfera da atenção básica à saúde, sobretudo no atendimento pré-natal e na assistência ao parto 10,11 .

Adicionalmente, entende-se que a melhora da qualidade das informações de registro das estatísticas vitais constitui etapa essencial no processo de redução da mortalidade infantil 12,13. A análise das irregularidades locais nos sistemas de informação proporciona não só a melhora da qualidade das estatísticas de registro, e, conseqüentemente, a estimação da mortalidade infantil, mas também possibilita evidenciar problemas que podem ser relacionados à mortalidade entre as crianças menores de um ano de idade, identificar municípios que necessitam de maio- 
Distribuição percentual (\%) da população de acordo com a adequação das informações vitais por dimensão avaliada segundo porte populacional e Grande Região. Brasil, 2000-2002

\begin{tabular}{|c|c|c|c|c|c|c|c|c|c|c|c|c|c|c|}
\hline & \multirow{3}{*}{ P } & \multirow{3}{*}{$\begin{array}{l}\text { Percentual da } \\
\text { população } \\
\text { total }\end{array}$} & \multicolumn{12}{|c|}{ Dimensão avaliada } \\
\hline & & & \multicolumn{3}{|c|}{ Mortalidade } & \multicolumn{3}{|c|}{ Natalidade } & \multicolumn{3}{|c|}{ Mal definidos } & \multicolumn{3}{|c|}{3 dimensões } \\
\hline & & & 1 & II & III & I & II & III & $\mathrm{I}$ & II & III & I & II & III \\
\hline \multicolumn{15}{|c|}{ População menor de } \\
\hline \multicolumn{15}{|c|}{50 mil habitantes } \\
\hline Norte & 5.701 .745 & 3,3 & 27,1 & 46,5 & 26,4 & 56,4 & 25,8 & 17,8 & 35,8 & 21,7 & 42,6 & 8,6 & 31,0 & 60,5 \\
\hline Nordeste & 24.078 .350 & 14,0 & 45,0 & 42,0 & 13,1 & 77,1 & 16,3 & 6,7 & 16,3 & 23,5 & 60,2 & 6,5 & 28,5 & 65,0 \\
\hline Sudeste & 17.387 .575 & 10,1 & 82,9 & 12,6 & 4,6 & 85,8 & 10,2 & 4,1 & 74,6 & 15,9 & 9,5 & 64,0 & 22,0 & 14,0 \\
\hline Sul & 10.724 .723 & 6,2 & 93,2 & 6,3 & 0,5 & 91,8 & 7,4 & 0,8 & 92,1 & 7,5 & 0,4 & 80,6 & 17,8 & 1,5 \\
\hline Centro-Oeste & 4.565 .973 & 2,7 & 78,8 & 19,1 & 2,1 & 86,7 & 11,6 & 1,7 & 91,4 & 7,6 & 1,0 & 67,7 & 27,7 & 4,6 \\
\hline Brasil & 62.458 .365 & 36,3 & 64,6 & 26,4 & 9,0 & 80,8 & 13,6 & 5,6 & 52,8 & 17,3 & 29,9 & 39,9 & 25,0 & 35,1 \\
\hline \multicolumn{15}{|c|}{ População com 50 mil } \\
\hline \multicolumn{15}{|c|}{ habitantes ou mais } \\
\hline Norte & 7.515 .033 & 4,4 & 63,5 & 20,6 & 15,9 & 75,8 & 14,7 & 9,5 & 47,6 & 29,7 & 22,8 & 33,1 & 39,3 & 27,6 \\
\hline Nordeste & 24.193 .682 & 14,1 & 52,8 & 35,2 & 12,0 & 78,2 & 11,7 & 10,1 & 45,3 & 28,9 & 25,8 & 31,6 & 35,2 & 33,2 \\
\hline Sudeste & 55.936 .483 & 32,5 & 87,2 & 11,8 & 1,0 & 93,0 & 6,0 & 1,0 & 91,5 & 8,1 & 0,4 & 75,7 & 22,2 & 2,0 \\
\hline Sul & 14.660 .396 & 8,5 & 80,3 & 19,7 & 0,0 & 81,6 & 17,1 & 1,3 & 92,9 & 7,1 & 0,0 & 61,2 & 37,5 & 1,3 \\
\hline Centro-Oeste & 7.273 .726 & 4,2 & 89,1 & 8,5 & 2,4 & 91,3 & 6,3 & 2,4 & 98,0 & 2,0 & 0,0 & 85,1 & 11,7 & 3,2 \\
\hline Brasil & 109.579 .320 & 63,7 & 77,2 & 18,4 & 4,4 & 86,9 & 9,3 & 3,7 & 78,9 & 13,6 & 7,5 & 61,7 & 27,6 & 10,7 \\
\hline \multicolumn{15}{|l|}{ População total } \\
\hline Norte & 13.216 .777 & 7,7 & 47,8 & 31,8 & 20,4 & 67,4 & 19,5 & 13,1 & 42,5 & 26,2 & 31,3 & 22,5 & 35,7 & 41,8 \\
\hline Nordeste & 48.272 .031 & 28,1 & 48,9 & 38,5 & 12,5 & 77,7 & 14,0 & 8,4 & 30,8 & 26,2 & 43,0 & 19,1 & 31,9 & 49,1 \\
\hline Sudeste & 73.324 .058 & 42,6 & 86,2 & 12,0 & 1,9 & 91,3 & 7,0 & 1,7 & 87,5 & 10,0 & 2,6 & 72,9 & 22,2 & 4,9 \\
\hline Sul & 25.385 .119 & 14,8 & 85,8 & 14,0 & 0,2 & 85,9 & 13,0 & 1,1 & 92,6 & 7,3 & 0,2 & 69,4 & 29,2 & 1,4 \\
\hline Centro-Oeste & 11.839 .700 & 6,9 & 85,1 & 12,6 & 2,3 & 89,5 & 8,3 & 2,2 & 95,5 & 4,1 & 0,4 & 78,4 & 17,9 & 3,7 \\
\hline Brasil & 172.037 .685 & 100,0 & 72,6 & 21,3 & 6,1 & 84,7 & 10,9 & 4,4 & 69,4 & 15,0 & 15,6 & 53,8 & 26,7 & 19,5 \\
\hline
\end{tabular}

* 54 municípios foram excluídos da análise por terem sido criados em 2001.

I: satisfatório; II: não satisfatório; III: deficiente.

res investimentos 14,15 e fornecer subsídios para a formulação de políticas públicas 16 . Neste contexto, a melhora dos sistemas de informação em saúde tem sido considerada como um dos desafios a ser enfrentado para alcance das metas do milênio na região das Américas 17.

Neste trabalho, procurou-se aperfeiçoar critérios estabelecidos anteriormente 5 , com base em sugestões de pesquisadores e de gestores do Ministério da Saúde, que utilizaram a referida metodologia para investigar a adequação das informações em municípios selecionados 18,19. As críticas se referiram, principalmente, ao uso dos mesmos critérios para os municípios de pequeno porte e de grande porte populacional, tendo em vista a flutuação temporal dos dados nos municípios pequenos, bem como a falta de critérios estatísticos para estabelecer os limites críticos para classificação do município como satisfatório, não satisfatório ou deficiente.
Na presente análise, levando-se em consideração as sugestões fornecidas, o método foi aperfeiçoado, estabelecendo-se critérios para dois grupos distintos: os municípios com $50 \mathrm{mil}$ habitantes ou mais e os com menos de $50 \mathrm{mil}$ habitantes. Além disso, os limites críticos para classificação da adequação das informações vitais foram estabelecidos com base na estimação estatística, por meio de limites de confiança para a média dos indicadores calculados nas UF com informações adequadas, segundo critérios estabelecidos pela RIPSA 6 .

É preciso destacar que os critérios de adequação foram bastante flexíveis, sobretudo entre os municípios pequenos, não só pela grande flutuação temporal dos indicadores devido ao tamanho populacional, como também para incentivar o uso das estatísticas vitais. Ressalva-se, porém, que essa flexibilidade pode acarretar, por vezes, no uso de informações inadequadas, com- 
Distribuição espacial dos municípios de acordo com a adequação das informações vitais por dimensão avaliada. Brasil, $2000-2002$.

1a) Mortalidade

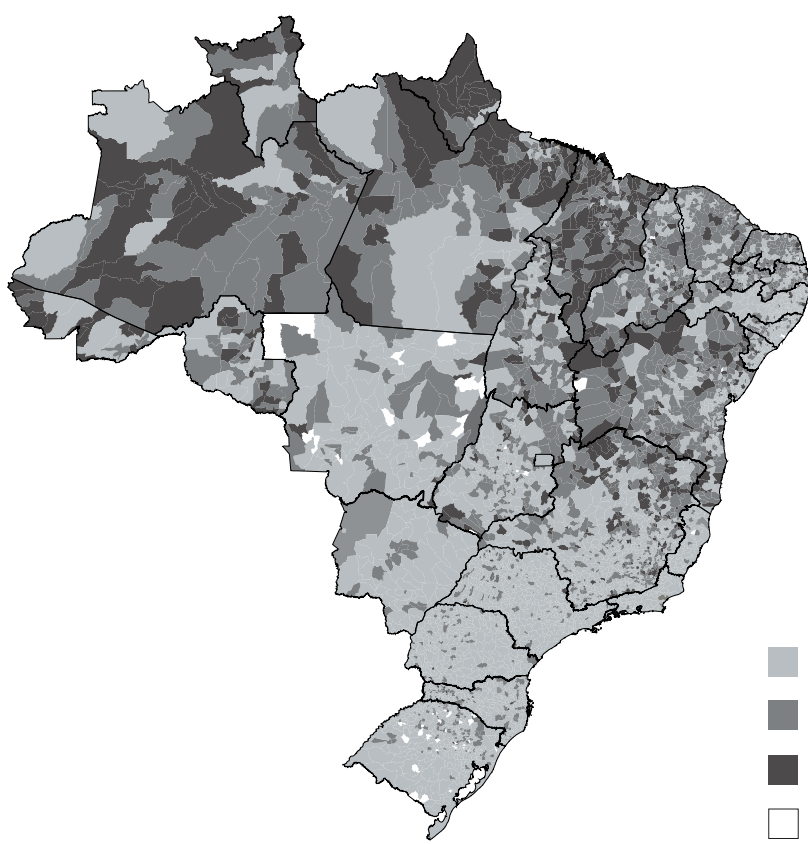

1b) Mal definidos

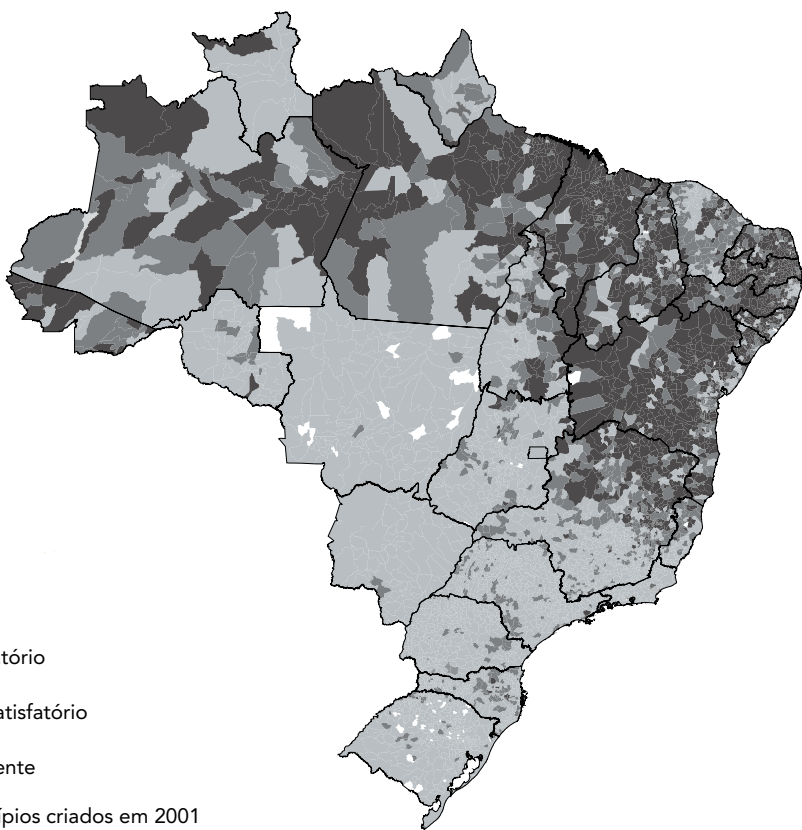

1c) Natalidade

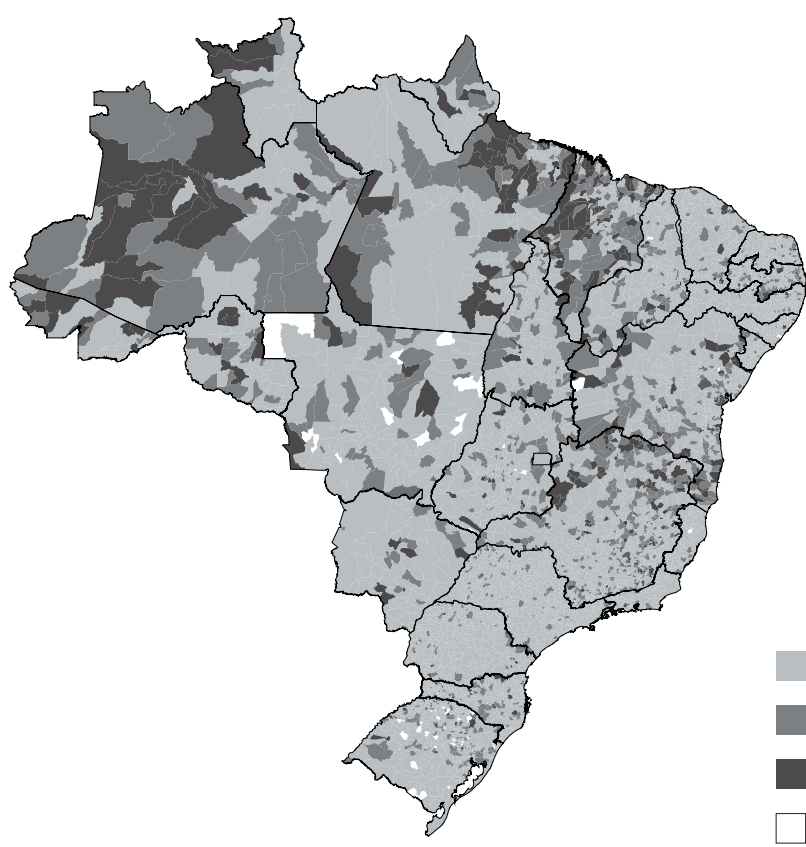

1d) 3 dimensões

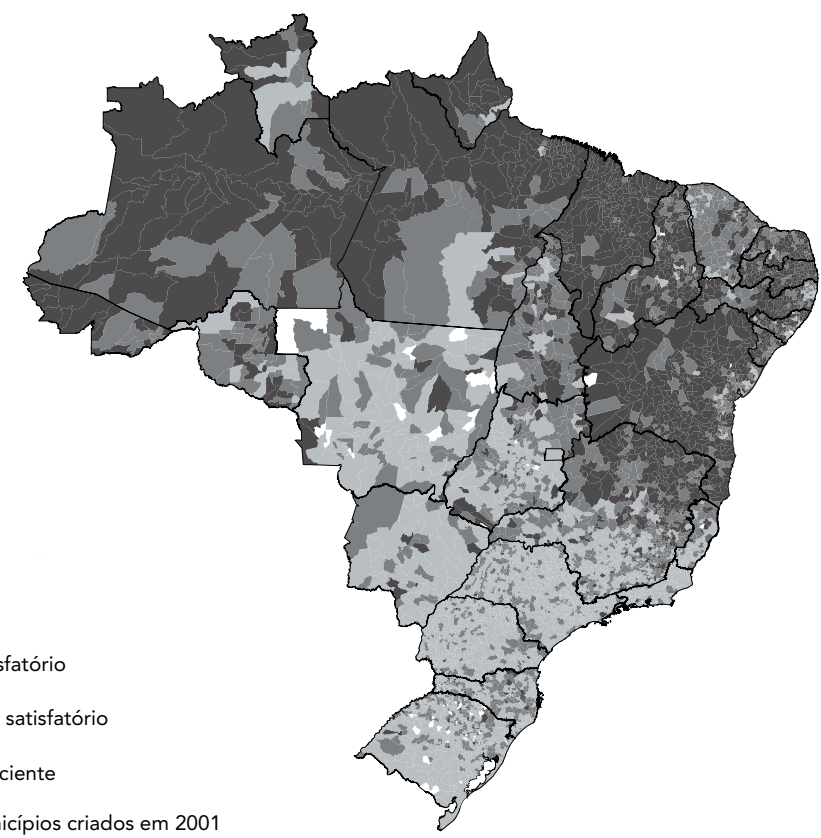


prometendo os indicadores de saúde construídos de modo direto.

Mudanças complementares foram feitas na definição do indicador relativo à proporção de mortes mal definidas, sendo considerados, desta feita, todos os óbitos sem definição da causa básica em vez de se restringir aos menores de um ano, bem como na utilização da razão entre nascidos vivos informados e estimados em vez da taxa bruta de natalidade, que apresenta variações regionais significativas.

Depreende-se dos resultados aqui apresentados que para, aproximadamente, $55 \%$ da população brasileira, a adequação das informações de nascimentos e óbitos pode ser considerada satisfatória. Embora a adequação das informações não signifique cobertura completa dos sistemas, para essa parcela da população, o monitoramento da mortalidade infantil pode ser realizado mediante o uso direto dos dados informados ao Ministério da Saúde.

Por outro lado, os resultados da presente análise enfatizam as desigualdades em saúde da população brasileira, que se refletem não apenas na variação dos níveis da mortalidade infantil, como já amplamente documentado 20,21,22, mas também na desigualdade de adequação das informações para o seu cálculo. Os problemas de estimação são mais presentes, justamente, nas áreas com as piores situações de saúde, que detêm maior precariedade das informações e necessitam de atenção específica.

Os achados mostram um gradiente regional importante: o percentual de adequação das estatísticas vitais, nos três aspectos considerados, mostrou contrastes relevantes entre as regiões Norte e Nordeste e o Centro-Sul. As desigualdades na adequação das informações ocorrem, igualmente, quando se comparam municípios de maior porte populacional com os municípios pequenos. Apesar de critérios bem mais flexíveis para esses últimos, os achados mostram, con- sistentemente, melhor informação nas cidades grandes.

De certa forma, esses achados são paradoxais, já que nos municípios pequenos ocorre baixo contingente de nascimentos, e menor ainda de óbitos infantis. Seria esperado, portanto, um maior controle da informação dos eventos vitais. Refletindo, por um lado, a iniqüidade no acesso aos programas e serviços de saúde, e por outro, o descaso com a informação como evidência da situação local de saúde entre as crianças menores de um ano, mostrou-se que apenas um terço dos municípios com menos de 50 mil habitantes tem grau satisfatório na informação dos dados vitais.

Dentre as limitações deste estudo, destaca-se que em, aproximadamente, $4 \%$ dos municípios com menos de 50 mil habitantes, localizados nas oito UF consideradas, consensualmente, como tendo adequação das informações, foram encontradas deficiências claras nas informações vitais. Embora o percentual tenha sido pequeno, isso pode ter afetado os limites estatísticos, resultando em critérios mais flexíveis ainda no grupo de municípios de pequeno porte populacional.

Em suma, em relação aos três aspectos considerados no estudo, o sistema de nascidos vivos é o que teve a melhor avaliação. No tocante à mortalidade, foram encontrados problemas tanto no que se refere à quantidade de óbitos informados, como também à completitude no preenchimento da causa básica, o que não permite estabelecer, adequadamente, o perfil de mortalidade em grande parte do território nacional.

Faz-se, assim, necessário não só reduzir a subnotificação de mortes em menores de um ano. É preciso também melhorar a qualidade do preenchimento da declaração de óbito, para que as informações possam orientar os programas de saúde voltados, especificamente, para a redução das iniqüidades da mortalidade infantil, que poderão ter maior efetividade mediante o conhecimento dos diferenciais e compreensão dos seus determinantes 23 . 


\section{Resumo}

Neste trabalho, analisam-se as desigualdades sócio-espaciais da adequação das informações de nascimentos (SINASC) e óbitos (SIM) do Ministério da Saúde para o cálculo da mortalidade infantil por município. A análise foi realizada de acordo com o porte populacional do município e região geográfica no período 2000 2002, considerando-se cinco indicadores: coeficiente geral de mortalidade padronizado por idade; razão entre nascidos vivos informados e estimados; desvio médio relativo do coeficiente de mortalidade; desvio médio relativo da taxa de natalidade; percentual de óbitos sem definição da causa básica de morte. Os critérios de adequação foram estabelecidos estatisticamente nas Unidades da Federação com informações consideradas adequadas. Os resultados mostram desigualdades sócio-espaciais importantes: o percentual de adequação é maior no Centro-Sul e entre os municípios de maior porte populacional. Em relação aos três aspectos estudados, o SINASC teve a melhor avaliação. Quanto ao SIM, além de reduzir a subnotificação, é preciso melhorar a qualidade do preenchimento da causa de óbito, para que as informações possam orientar adequadamente os programas de saúde voltados para a redução das iniqüidades da mortalidade infantil no Brasil.

Sistemas de Informação; Mortalidade Infantil; Indicadores

\section{Colaboradores}

C. L. T. Andrade trabalhou na análise dos dados, definiu o conteúdo, elaborou tabelas e mapas e redigiu a principal parte do artigo. C. L. Szwarcwald participou na metodologia, na concepção do artigo e colaborou na redação do mesmo.

\section{Referências}

1. Brass W. Demographic data analysis in less developed countries: 1946-1996. Population Studies 1996; 50:451-67.

2. Centro Latinoamericano de Demografía. Estimaciones y proyecciones de población en los países de América Latina 1950-2050. Santiago: Centro Latinoamericano de Demografía; 1996. (Boletín Demográfico, 58).

3. Pujol JM. Nuevas metodologías para evaluar y ajustar datos demográficos. Notas Poblac 1985; 13:57-73.

4. Guzman JM. Some problems concerning the selection of the most appropriate mortality model for the indirect estimation of infant mortality. Notas Poblac 1985; 13:75-103.

5. Szwarcwald CL, Leal MC, Andrade CLT, Souza Jr. PRB. Estimação da mortalidade infantil no Brasil: o que dizem as informações de óbitos e nascimentos do Ministério da Saúde? Cad Saúde Pública 2002; 18:1725-36.
6. Rede Interagencial de Informações para a Saúde. Indicadores básicos de saúde no Brasil: conceitos e aplicações. Brasília: Organização Pan-Americana da Saúde; 2002.

7. Simões CCS, Monteiro CA. Tendência secular e diferenças regionais da mortalidade infantil no Brasil. In: Monteiro CA, organizador. Velhos e novos males da saúde no Brasil: a evolução do país e suas doenças. São Paulo: Editora Hucitec/Núcleo de Pesquisas Epidemiológicas em Nutrição e Saúde, Universidade de São Paulo; 1995. p. 153-6.

8. Guimarães ZA, Costa MCN, Paim JS, Silva LMV. Decline and social inequalities of infant mortality caused by diarrhea. Rev Soc Bras Med Trop 2001; 34:473-8.

9. Leal MC, Szwarcwald CL. Evolução da mortalidade neonatal no Estado do Rio de Janeiro, Brasil, de 1979 a 1993. 1 - Análise por grupo etário segundo região de residência. Rev Saúde Pública 1996; 30:403-12. 
10. Barros F, Victora C, Barros A, Santos I, Albernaz E, Matijasevich A, et al. The challenge of reducing neonatal mortality in middle-income countries: findings from three Brazilian birth cohorts in 1982, 1993, and 2004. Lancet 2005; 365:847-54.

11. Leal MC, Gama SGN, Campos MR, Cavalini LT, Garbayo LS, Brasil CLP, et al. Fatores associados à morbi-mortalidade perinatal em uma amostra de maternidades públicas e privadas do Município do Rio de Janeiro, 1999-2001. Cad Saúde Pública 2004; 20 Suppl 1:S20-33.

12. Gould JB, Chavez G, Marks AR, Liu H. Incomplete birth certificates: a risk marker for infant mortality. Am J Public Health 2002; 92:79-81.

13. de Meer K, Bergman R, Kusner JS. Socio-cultural determinants of child mortality in southern Peru: including some methodological considerations. Soc Sci Med 1993; 36:317-31.

14. Neumann NA, Victora CG, Halpern R, Guimarães PR, Cesar JA. Assessment of the performance of Pastoral da Criança, a health support group, in promoting child survival and health education in Criciúma, a city in Southern Brazil. Rev Panam Salud Pública 1999; 5:400-10.

15. Whitman S, Silva A, Shah A, Ansell D. Diversity and disparity: GIS and small-area analysis in six Chicago neighborhoods. J Med Syst 2004; 28:397-411.

16. Lumbiganon P, Panamonta M, Laopaiboon M, Pothinam S, Patithat N. Why are Thai official perinatal and infant mortality rates so low? Int J Epidemiol 1990; 19:997-1000.

17. Torres C, Mujica OJ. Health, equity, and the millennium development goals. Rev Panam Salud Pública $2004 ; 15: 430-9$.
18. Secretaria de Vigilância em Saúde, Ministério da Saúde. Monitoramento da acurácia dos Sistemas de Informações sobre Mortalidade e Nascidos Vivos. In: Secretaria de Vigilância em Saúde, Ministério da Saúde, organizador. Anais da 3a EXPOEPI - Mostra Nacional de Experiências Bem-sucedidas em Epidemiologia, Prevenção e Controle de Doenças. Brasília: Ministério da Saúde; 2004. p. 173-80.

19. Pinheiro AMCM. Avaliação dos sistemas de informação de nascidos vivos e de mortalidade para a obtenção da mortalidade neonatal em Ilhéus, Bahia [Tese de Doutorado]. São Paulo: Universidade de São Paulo; 2003.

20. Andrade CLT, Szwarcwald CL, Gama SGN, Leal MC. Desigualdades sócio-econômicas do baixo peso ao nascer e da mortalidade perinatal no Município do Rio de Janeiro, 2001. Cad Saúde Pública 2004; 20 Suppl 1:S44-51.

21. Sastry N. Trends in socioeconomic inequalities in mortality in developing countries: the case of child survival in São Paulo, Brazil. Demography 2004; 41:443-64.

22. Schneider MC, Castillo-Salgado C, Loyola-Elizondo E, Bacallao J, Mujica OJ, Vidaurre M, et al. Trends in infant mortality inequalities in the Americas: 1955-1995. J Epidemiol Community Health 2002; 56:538-41.

23. Muller M, Drack G, Schindler C, Bucher HU. Liveborn and stillborn very low birthweight infants in Switzerland: comparison between hospital based birth registers and the national birth register. Swiss Med Wkly 2005; 135:433-9.

Recebido em 08/Dez/2005

Versão final reapresentada em 19/Set/2006

Aprovado em 20/Out/2006 